\title{
Correction to: A High-Precision and Miniature Fiber Bragg Grating-Based Force Sensor for Tissue Palpation During Minimally Invasive Surgery
}

\author{
Changhu Lv, Shuxin Wang, and Chaoyang Shi
}

Key Laboratory of Mechanism Theory and Equipment Design of Ministry of Education, School of Mechanical Engineering, Tianjin University, Tianjin 300072, China

Correction to: Annals of Biomedical Engineering ( $\subset$ 2019) https://doi.org/10.1007/s10439-019-02388-w

The second equation in the section "Definition of Objective Functions and Constraints" was corrected to fix a mismatch between the PDF and HTML versions of the article.

Publisher's Note Springer Nature remains neutral with regard to jurisdictional claims in published maps and institutional affiliations.

Address correspondence to Chaoyang Shi, Key Laboratory of Mechanism Theory and Equipment Design of Ministry of Education, School of Mechanical Engineering, Tianjin University, Tianjin 300072, China. Electronic mail: chaoyang_shi@tju.edu.cn

The original article can be found online at https://doi.org/10. 1007/s10439-019-02388-w. 